\title{
Patterned Growth of Vertically Aligned ZnO Nanowire Arrays on Inorganic Substrates at Low Temperature without Catalyst
}

\author{
Sheng Xu,${ }^{\dagger}$ Yaguang Wei, ${ }^{\dagger}$ Melanie Kirkham, ${ }^{\dagger}$ Jin Liu, ${ }^{\dagger}$ Wenjie Mai, ${ }^{\dagger}$ Dragomir Davidovic,,${ }^{\ddagger}$ \\ Robert L. Snyder, ${ }^{\dagger}$ and Zhong Lin Wang ${ }^{*, \dagger}$ \\ School of Material Science and Engineering, School of Physics, Georgia Institute of Technology, \\ Atlanta, Georgia 30332
}

Received September 2, 2008; E-mail: zhong.wang@mse.gatech.edu

\begin{abstract}
Vertically aligned $\mathrm{ZnO}$ nanowires (NWs) have a variety of astonishing applications in electronics as well as optoelectronic and electromechanical nanodevices, such as solar cells, ${ }^{1}$ field emission devices, ${ }^{2,3}$ UV lasers, ${ }^{4,5}$ light emitting diodes, ${ }^{6}$ and piezo-nanogenerators. ${ }^{7,8}$ Growth of aligned $\mathrm{ZnO}$ NWs has been achieved on $\mathrm{GaN}$ via physical vapor deposition (PVD) at a high temperature of $500-800{ }^{\circ} \mathrm{C}$, with the use of gold as a catalyst, ${ }^{9}$ in which the catalyst initiated and guided the growth, and the epitaxial orientation relationship between the $\mathrm{ZnO}$ NWs and the GaN substrate led to the aligned growth. This strategy may have the risk of introducing catalyst residual atoms into the $\mathrm{ZnO} \mathrm{NWs}$, which is incompatible with silicon technology. Patterned growth of aligned $\mathrm{ZnO}$ NWs has also been accomplished by PVD using nanosphere-masking lithography on sapphire, ${ }^{10,11}$ which was conducted at high growth temperature, and therefore was not compatible with many other materials. Metal-organic vapor-phase epitaxial growth ${ }^{12}$ and template-assisted growth ${ }^{13}$ have also been employed for the fabrication of vertically aligned $\mathrm{ZnO} \mathrm{NWs}$. Yi et al. have demonstrated a technique of growing periodically arranged $\mathrm{ZnO}$ nanorod arrays on a silicon substrate coated with $\mathrm{ZnO}$ seeds by electron beam lithography (EBL). ${ }^{14}$ But their nanorods were thick in diameter and had a rather small aspect ratio, ${ }^{15}$ which greatly limited their applications.
\end{abstract}

To grow high-quality $\mathrm{ZnO} \mathrm{NW}$ arrays, we need an approach that can meet the following three requirements. First, the growth has to be at low temperature so that the NWs can be integrated with general substrates. Second, the NWs have to be grown following a designed pattern, with a high degree of control in size, orientation, dimensionality, uniformity, and possibly shape. Finally, the catalyst may need to be eliminated for integration with silicon based technology. In this paper, we demonstrate an approach that meets all of these requirements. Our method combines EBL and a low temperature hydrothermal method to achieve patterned and aligned growth of $\mathrm{ZnO} \mathrm{NWs}$ at $<100{ }^{\circ} \mathrm{C}$ on general inorganic substrates, such as $\mathrm{Si}$ and $\mathrm{GaN}$, without using a catalyst. This approach opens up the possibility of creating patterned, vertically aligned one-dimensional $\mathrm{ZnO}$ nanostructures for many applications such as sensor arrays, piezoelectric antenna arrays, two-dimensional photonic crystals, integrated circuit (IC) interconnects, and nanogenerators.

Detailed experimental methods are described in the Supporting Information (SI). The nonepitaxial growth of $\mathrm{ZnO} \mathrm{NW}$ arrays on Si wafers (or any other inorganic substrates) was assisted by a thin film of polycrystalline $\mathrm{ZnO}$ seeds. EBL was employed to generate a patterned mask, which was made of poly(methyl methacrylate) (PMMA), over the $\mathrm{ZnO}$ seed film. The hydrothermal growth

School of Material Science and Engineering.

\$ School of Physics.

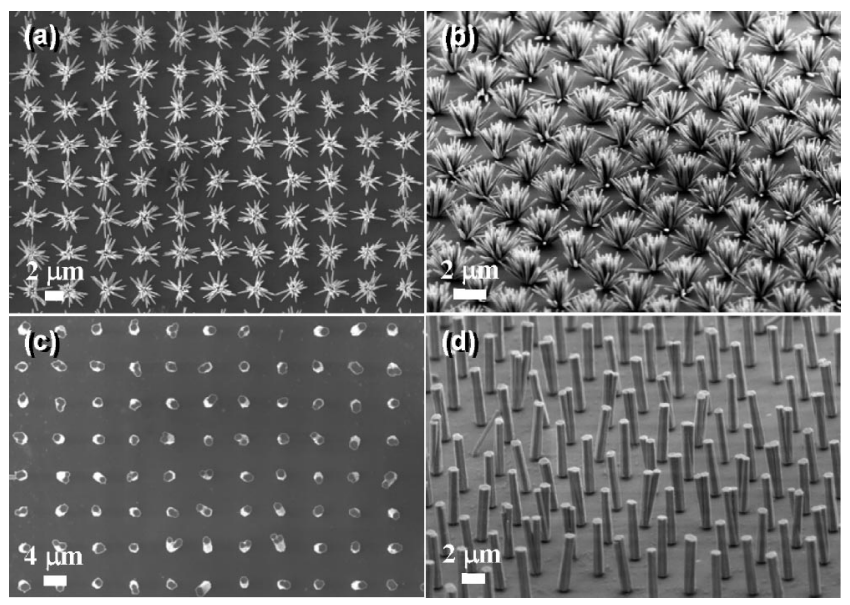

Figure 1. (a) Top view and (b) $60^{\circ}$ tilt view of the $\mathrm{ZnO} \mathrm{NW}$ arrays on a $\mathrm{Si}$ wafer grown at $70^{\circ} \mathrm{C}$. (c) Top view and (d) $60^{\circ}$ tilt view of the $\mathrm{ZnO}$ $\mathrm{NW}$ arrays on a $\mathrm{Si}$ wafer grown at $95^{\circ} \mathrm{C}$.

mechanism has been well understood and documented. ${ }^{16-18}$ One thing worth noting is that during the hydrothermal growth process, by virtue of the solution surface tension, the substrate was put facedown floating on the nutrient solution surface (Figure S1 in the SI), to keep any precipitates from falling down onto the substrate from the solution body, which would otherwise inhibit the growth of the desired nanostructures or possibly initiate secondary growth. Even though the substrate was annealed at $300{ }^{\circ} \mathrm{C}$ for $20 \mathrm{~min}$, the $\mathrm{ZnO}$ seed film on the substrate was still composed of many tiny crystals with random in-plane orientations, ranging from several nanometers to tens of nanometers in size (see SI Figure S2). The sizes of the EBL patterned openings in this study were all greater than $100 \mathrm{~nm}$, in which a number of $\mathrm{ZnO}$ grains were exposed. Consequently, in most cases, multiple NWs would grow out of one single spot, as illustrated in Figure 1a,b. Under appropriate conditions, $\mathrm{ZnO} \mathrm{NWs}$ in close proximity are inclined to have coalescence effects. ${ }^{19,20}$ In our experiments, by increasing the growth temperature from $70{ }^{\circ} \mathrm{C}$ to a relatively high growth temperature $\left(95^{\circ} \mathrm{C}\right)$, those multiple NWs from one single opening merged together, resulting in a thicker NW as shown in Figure 1c,d (see SI Figure S3 for half-merged NWs).

The epitaxial growth of aligned $\mathrm{ZnO} \mathrm{NW}$ arrays on Si doped n-type $\mathrm{GaN}$ on a sapphire substrate ${ }^{21}$ was performed in an analogous way as on a Si wafer. The lattice mismatch between the wurzite $\mathrm{GaN}$ (0001) plane and wurzite $\mathrm{ZnO}(0001)$ plane is $1.8 \%{ }^{22}$ which led to a perfectly aligned growth of the $\mathrm{ZnO} \mathrm{NW}$ array, as shown in Figure 2a,b. Because the as-grown NWs have a small diameter and a large aspect ratio, the sample needs a supercritical drying when the hydrothermal growth is complete. ${ }^{23}$ Or else, when 


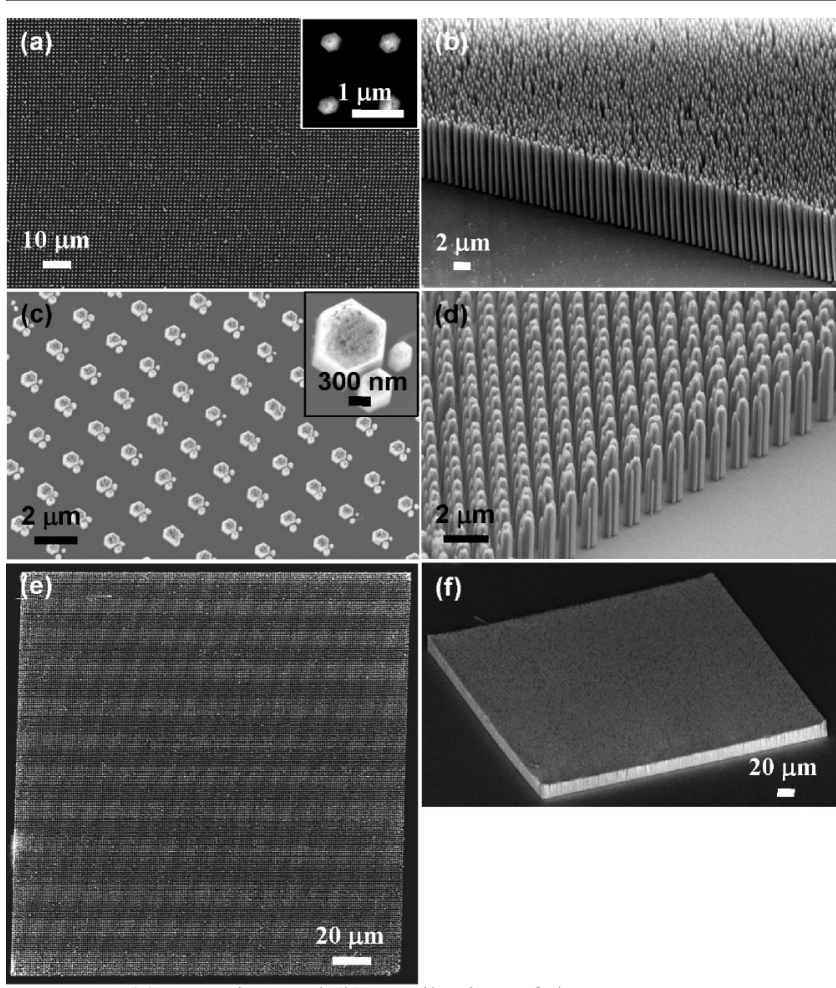

Figure 2. (a) Top view and (b) $60^{\circ}$ tilt view of the $\mathrm{ZnO} \mathrm{NW}$ arrays on a GaN substrate. (c) Top view and (d) $60^{\circ}$ tilt view of the NWs growing out of 400, 200, and $100 \mathrm{~nm}$ sized holes. Insets are enlarged top view image. Insets are enlarged top view of the NWs. (e) Top view and (f) $60^{\circ}$ tilt view of a $200 \mu \mathrm{m} \times 200 \mu \mathrm{m}$ patterned NW arrays.

the liquid droplets on the NW arrays get evaporated and shrink in size, the surface tension of the droplet would sweep all the vertical NWs down onto the substrate.

An interesting phenomenon was noticed when we compared the EBL pattern before hydrothermal growth with that after hydrothermal growth (see SI Figure S4). Out of $100 \mathrm{~nm}$ sized holes the grown NWs were $300 \mathrm{~nm}$ in diameters. The width of the NWs was three times the diameter of the holes. We proposed a two-step growing process for the ZnO NWs as illustrated in Figure S5 in the SI. In the first step, the NW grew out of the photoresist hole with the same lateral dimension as confined by the hole. Then in the following step, there was no lateral confinement for it, and the NW could grow both vertically and laterally, but apparently faster in the vertical direction than in the lateral direction.

Even though the NWs would expand laterally when they grew out of the confining holes, we could still tune the width of the NWs in a very responsive and reliable fashion. As shown in Figure 2c,d, when we made an array of circles with 400, 200, and $100 \mathrm{~nm}$ in diameter, respectively, the developed NWs showed proportional sizes to the holes accordingly. In addition, the pitch between the roles and columns of the pattern could also be straight forwardly varied for different application purposes.

The crystal structure and vertical alignment of the as-grown $\mathrm{ZnO}$ NW arrays were examined by X-ray diffraction (XRD) pattern and rocking curve measurements. The $\theta-2 \theta$ scanning results of the sample are shown in Figure 3, where the main peaks have been labeled. In the inset of Figure 3 is the $\theta$-rocking curve for the peak at $34.393^{\circ}$ of the $\mathrm{ZnO}(002)$ plane. It exhibits a full width at halfmaximum value of $0.15^{\circ}$, which indicates almost perfect vertical alignment of the $\mathrm{ZnO}$ NWs on the GaN substrate.

In summary, we have developed a low temperature hydrothermal growth method to fabricate patterned and vertically aligned $\mathrm{ZnO}$

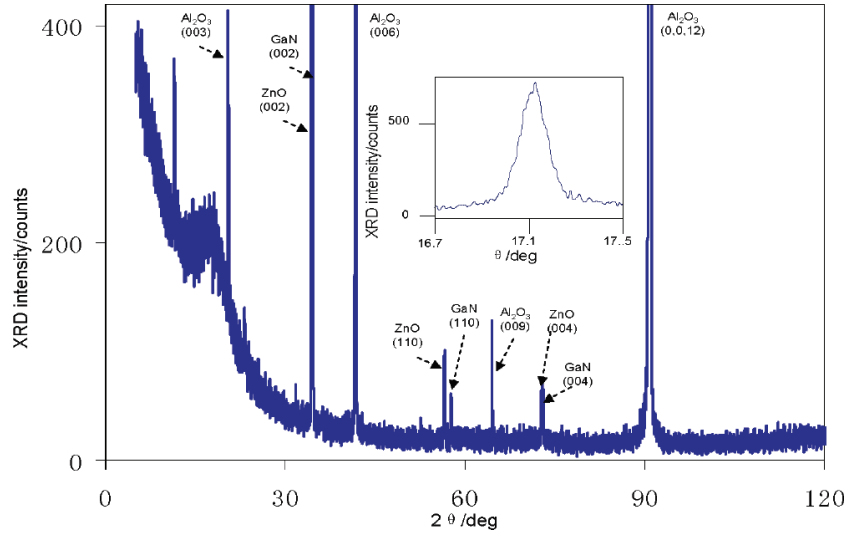

Figure 3. $\mathrm{X}$-ray diffraction pattern of the as-grown $\mathrm{ZnO}$ nanowire arrays on $\mathrm{GaN}$ (001). Inset is the $\mathrm{ZnO}$ (002) peak rocking curve.

NW arrays on a general inorganic substrate that may or may not have an epitaxial orientation relationship with $\mathrm{ZnO}$. On nonepitaxial substrates coated with $\mathrm{ZnO}$ seed film, we could grow $\mathrm{ZnO} \mathrm{NW}$ arrays with one NW on one site by increasing the growth temperature. On epitaxial substrates, perfectly aligned highly uniform $\mathrm{ZnO} \mathrm{NW}$ arrays could be grown with controllable NW size and tunable pattern pitch. It could also be easily scaled up, as shown in Figure 2e,f.

Acknowledgment. This research was supported by BES DOE, NSF, and Emory-Georgia Tech CCNE funded by the NIH.

Supporting Information Available: Experimental details about the synthesis and characterization of the nanowires arrays. This material is available free of charge via the Internet at http://pubs.acs.org.

\section{References}

(1) Law, M.; Greene, L. E.; Johnson, J. C.; Saykally, R.; Yang, P. D. Nat. Mater. 2005, 4, 455.

(2) Bai, X. D.; Wang, E. G.; Gao, P. X.; Wang, Z. L. Nano Lett. 2003, 3, 1147.

(3) Wang, X. D.; Zhou, J.; Lao, C. S.; Song, J. H.; Xu, N. S.; Wang, Z. L. Adv. Mater. 2007, 19, 1627.

(4) Yang, P. D.; Yan, H. Q.; Mao, S.; Russo, R.; Johnson, J.; Saykally, R.; Morris, N.; Pham, J.; He, R. R.; Choi, H. J. Adv. Funct. Mater. 2002, 12 , 323.

(5) Huang, M. H.; Mao, S.; Feick, H.; Yan, H. Q.; Wu, Y. Y.; Kind, H.; Weber, E.; Russo, R.; Yang, P. D. Science 2001, 292, 1897.

(6) Lim, J. H.; Kang, C. K.; Kim, K. K.; Park, I. K.; Hwang, D. K.; Park, S. J. Adv. Mater. 2006, 18, 2720.

(7) Wang, Z. L.; Song, J. H. Science 2006, 312, 242

(8) Wang, X.; Song, J.; Liu, J.; Wang, Z. L. Science 2007, 316, 102.

(9) Park, H. K.; Oh, M. H.; Kim, S. W.; Kim, G. H.; Youn, D. H.; Lee, S.; Kim, S. H.; Kim, K. C.; Maeng, S. L. Etri J. 2006, 28, 787.

(10) Wang, X. D.; Summers, C. J.; Wang, Z. L. Nano Lett. 2004, 4, 423.

(11) Liu, D. F.; Xiang, Y. J.; Wu, X. C.; Zhang, Z. X.; Liu, L. F.; Song, L.; Zhao, X. W.; Luo, S. D.; Ma, W. J.; Shen, J.; Zhou, W. Y.; Wang, G.; Wang, C. Y.; Xie, S. S. Nano Lett. 2006, 6, 2375.

(12) Park, W. I.; Yi, G. C.; Kim, M.; Pennycook, S. J. Adv. Mater. 2002, 14, 1841.

(13) Liu, C. H.; Zapien, J. A.; Yao, Y.; Meng, X. M.; Lee, C. S.; Fan, S. S.; Lifshitz, Y.; Lee, S. T. Adv. Mater. 2003, 15, 838.

(14) Yong-Jin, K.; Chul-Ho, L.; Young Joon, H.; Gyu-Chul, Y.; Sung Soo, K.; Hyeonsik, C. Appl. Phys. Lett. 2006, 89, 163128.

(15) Jingbiao Cui, a. U. G. Nanotechnology 2007, 18, 15532

(16) Zhang, J.; Sun, L. D.; Yin, J. L.; Su, H. L.; Liao, C. S.; Yan, C. H. Chem. Mater. 2002, 14, 4172.

(17) Vayssieres, L. Adv. Mater. 2003, 15, 464

(18) Greene, L. E.; Law, M.; Goldberger, J.; Kim, F.; Zhang, Y. F.; Saykally, R. J.; Yang, P. D. Angew. Chem., Int. Ed. 2003, 42, 3031.

(19) Gupta, V.; Bhattacharya, P.; Yuzuk, Y. I.; Sreenivas, K.; Katiyar, R. S. J. Cryst. Growth 2006, 287, 39.

(20) Yang, R. S.; Wang, Z. L. Solid State Commun. 2005, 134, 741.

(21) Le, H. Q.; Chua, S. J.; Loh, K. P.; Fitzgerald, E. A.; Koh, Y. W. Nanotechnology 2006, 17, 483 .

(22) Wang, X. D.; Song, J. H.; Wang, Z. L. J. Mater. Chem. 2007, 17, 711.

(23) Liang, Y.; Zhen, C.; Zou, D.; Xu, D. J. Am. Chem. Soc. 2004, 126, 16338.

JA806952J 